\title{
Orbital Varix Masquerading as an Intraorbital Lymphoma
}

\author{
Jonathan L. Go, DO; Vivian Fasula, MD; and Igor Sirotkin, MD
}

\begin{abstract}
Clinical context was paramount to the diagnosis and management of a patient with periorbital pain and a history of systemic lymphoma.
\end{abstract}

W e present a case of an orbital varix masquerading as an orbital lymphoma. Our case underscores the importance of clinical correlation and thorough study of the ordered films by the ordering health care provider.

\section{CASE PRESENTATION}

An 84-year-old female veteran presented to the Bay Pines Veterans Affairs Healthcare System emergency department. She had a past ocular history of nonproliferative diabetic retinopathy in both eyes (OU) and senile cataracts OU. She had a complicated medical history most notable for congestive heart failure and Stage IV B cell follicular lymphoma, having received 6 rounds of chemotherapy, and has since been on rituximab maintenance therapy for the past few years.

The patient reported dyspnea on exertion, 30-pound weight gain, and ocular pain in her right eye (OD), more so than her left eye (OS) that was severe enough to wake her from sleep. She endorsed an associated headache but reported no visual loss or any other ocular symptoms other than conjunctival injection. On examination, the patient demonstrated jugular venous distension. X-ray imaging obtained in the emergency department demonstrated bilateral pleural effusions. Our patient was admitted subsequently for an exacerbation of congestive heart failure. She was monitored for euvolemia and discharged 4 days later.

During admission, imaging of the orbits was obtained. Computed tomography (CT) of the head without contrast demonstrated at least 4 intraorbital masses in the right orbit, measuring up to $22 \mathrm{~mm}$ in maximum diameter and at least 3 intraorbital masses in the left orbit, measuring up to $16 \mathrm{~mm}$ in di- ameter (Figure 1). Magnetic resonance imaging (MRI) with contrast of the brain and orbits was ordered, which demonstrated multiple bilateral uniformly enhancing, primarily extraconal masses present in both orbits, the largest of which occupied the superomedial aspect of the right orbit and measured $12 \times 18 \times 20 \mathrm{~mm}$. Further, the ophthalmic veins were noted to be engorged. The cavernous did not demonstrate any thrombosis. No other ocular structures were compromised, although there was compression of the extraocular muscles in both orbits (Figures 2, $3,4,5$, and 6). At that time, the reading radiologist suggested the most likely diagnosis was metastatic orbital lymphoma given the clinical history, which became the working diagnosis.

A few days after admission, the patient received an ophthalmic evaluation at the eye clinic. Visual acuity (VA) at this time was 20/200 that pinholed (PH) 20/70 OD and 20/30 without pinhole improvement OS. Refraction was $-2.50+1.50 \times 120$ OD and $-0.25+0.50 \times 065$ OS, which yielded visual acuities of 20/60 and 20/30, respectively. There was no afferent pupillary defect and pupils were symmetric. Goldmann tonometry demonstrated pressures of $11 \mathrm{~mm}$ of mercury OU at 1630. Slit-lamp and dilated fundus examinations were within normal limits except for $2+$ nuclear sclerotic cataracts, large cups of $0.6 \mathrm{OD}$ and $0.7 \mathrm{OS}$, and a mild epiretinal membrane OD. The decision was made to refer the patient to oculoplastic service for biopsy of the lesion to rule out a metastatic lymphoid solid tumor. At this juncture, the working diagnosis continued to be metastatic orbital lymphoma.

The patient underwent right anterior orbitotomy. Intraoperatively, after dissection to
Jonathan Go was an Ophthalmology Resident at the time the article was written, Vivian Fasula is an Opthalomologist, and Igor Sirotkin is a Neuroradiologist, all at Bay Pines Veterans Affairs Healthcare System in Florida. Igor Sirotkin is an Assistant Professor of Radiology at the University of Southern Florida in Tampa. Correspondence: Igor Sirotkin

(igor.sirotkin@va.gov)

Fed Pract. 2021;38(9). Published online September 15. doi:10.12788/fp.0182 
FIGURE 1 Noncontrast Head Computed Tomography
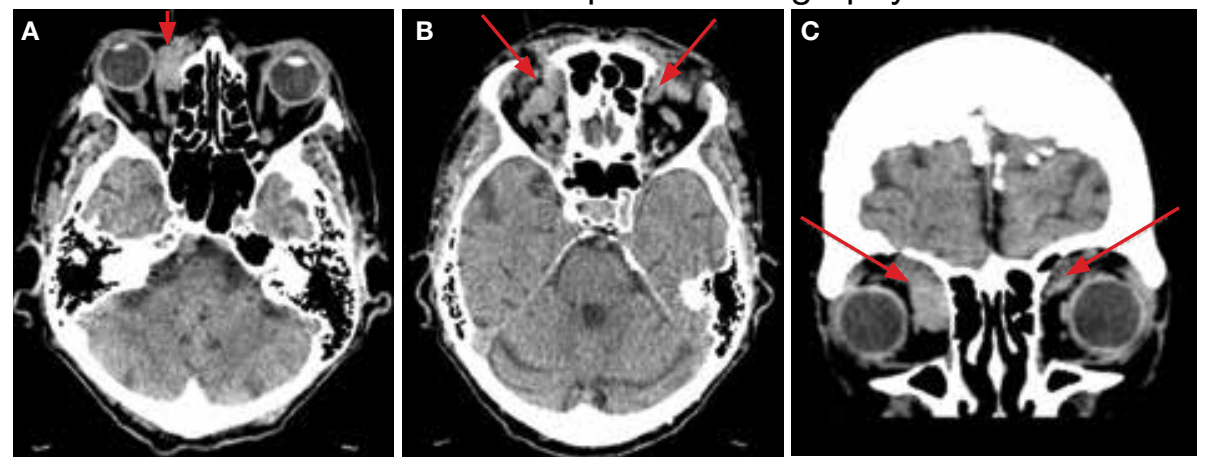

Images show extraconal intraorbital masses (arrows); A and B, axial view; C, coronal reformatted view.

FIGURE 2 T2-Weighted Fat Suppressed Magnetic Resonance Image Orbits
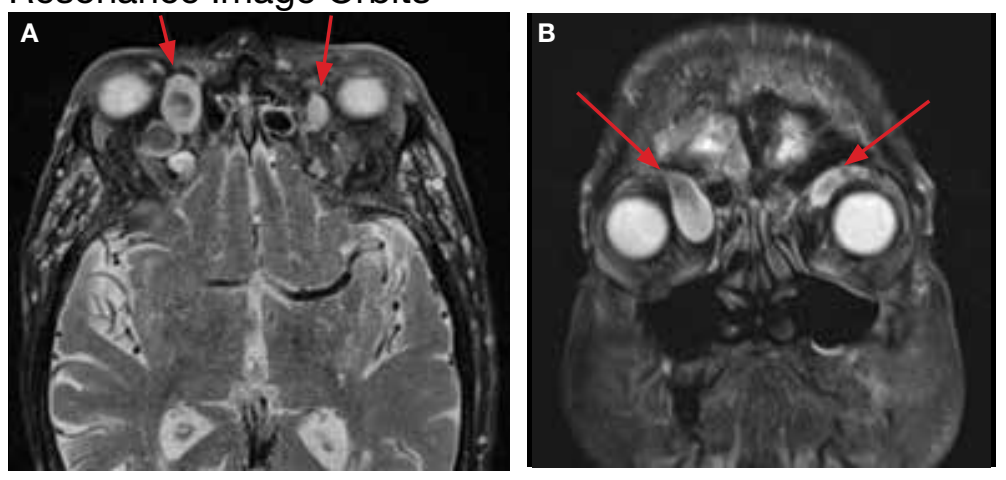

Images showing increased T2 signal with some partial decreased T2 signal centrally corresponding to the extraconal masses (arrows); A, axial; B, coronal.

\section{FIGURE 3 Axial T1 MPRAGE Postcontrast Brain Magnetic Resonance Image}
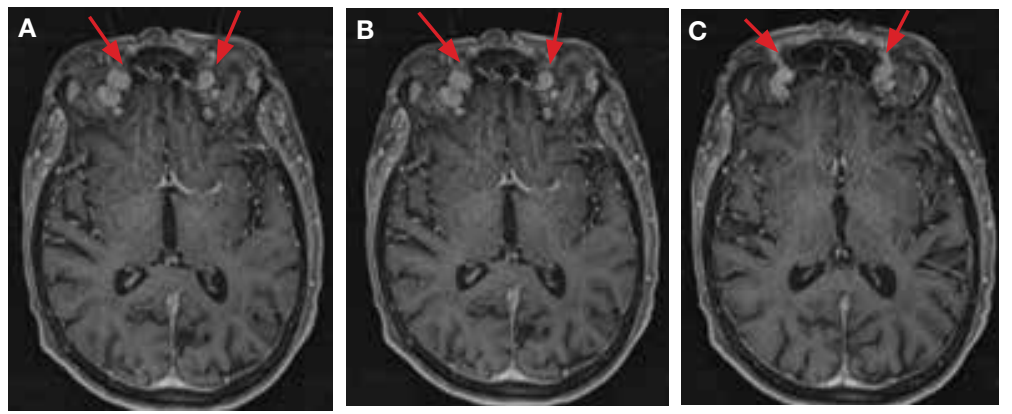

Abbreviation: MPRAGE, magnetization-prepared rapid acquisition and multiple gradient echoes.

Images show a dilated tortous superior ophthalmic vein (arrows) communicating superiorly with the supraorbital vein.

the lesion was accomplished, it was noted that the mass displayed a blue to purple hue consistent with a vascular malformation. It was decided to continue careful dissection instead of obtaining a biopsy. Continued dissection further corroborated a vascular lesion. Meticulous hemostasis was maintained during the dissection; however, dissection was halted after about 35-mm depth into the orbit, given concern for damaging the optic nerve. The feeding vessel to the lesion was tied off with two 5-0 vicryl sutures, and the specimen was cut distal to the ligation. During the procedure, pupillary function was continually checked. The rest of the surgery proceeded without any difficulty, and the specimen was sent off to pathology.

Pathology returned as an orbital varix with no thrombosis or malignant tissue. Surgery to remove lesions of the left orbit was deferred given radiologic findings consistent with vascular lesions, similar to the removed lesion from the right orbit. The patient is currently without residual periorbital pain after diuresis, and the patient's oncological management continues to be maintenance rituximab. The remaining lesions will be monitored with yearly serial imaging.

\section{DISCUSSION}

In a study of 242 patients, Bacorn and colleagues found that a clinician's preoperative assessment correlated with histopathologic diagnosis in $75.7 \%$ of cases, whereas the radiology report was correct in only $52.4 \%$ of cases. ${ }^{1}$ Retrospective analysis identified clues that could have been used to more rapidly elucidate the true diagnosis for our patient.

In regard to symptomatology, orbital varices present with intermittent proptosis, vision loss, and rarely, periorbital pain unless thrombosed. ${ }^{2,3}$ The severity of periorbital pain experienced by our patient is atypical of an orbital varix especially in the absence of a phlebolith. A specific feature of orbital varix is enlargement with the Valsalva maneuver. ${ }^{3}$ Although the patient did not report the notedsymptoms, more pointed questioning may have helped elucidate our patient's true diagnosis sooner.

Radiologically, the presence of a partial flow void (decreased signal on T2) is useful for confirming the vascular nature of a lesion as was present in our case. Specific to the radiologic evaluation of orbital varices, it is recommended to obtain imaging 
with and without the Valsalva maneuver. ${ }^{4}$ Ultrasound is a superb tool in our armamentarium to image orbital lesions. B-scan ultrasound with and without Valsalva should be able to demonstrate variation in size when standing (minimal distension) vs lying flat with Valsalva (maximal distension). ${ }^{4}$ Further, Doppler ultrasound would be able to demonstrate changes in flow within the lesion when comparing previously mentioned maneuvers. ${ }^{4}$ Orbital lymphoma would not demonstrate this variation.

The size change of an orbital varix lesion may be further demonstrated on head CT with contrast. On CT, an orbital varix will demonstrate isodensity to other venous structures, whereas orbital lymphomas will be hyperdense when compared to extraocular muscles. ${ }^{4,5}$ Further, a head CT without contrast may demonstrate phleboliths within an orbital varix. ${ }^{4}$ MRI should be performed with the Valsalva maneuver. On T1 and $\mathrm{T} 2$ studies, orbital varices demonstrate hypointensity when compared to extraocular muscles (EOMs). ${ }^{4}$ Lymphomas demonstrate a very specific radiologic pattern on MRI. On T1, they demonstrate isointensity to hypointensity when compared to EOMS, and on T2, they demonstrate iso- to hyperintensity when compared to EOMs. ${ }^{5}$ With respect to fluorodeoxyglucose (FDG) positron emission tomography (PET), our patient's orbital lesion did not demonstrate FDG uptake. In patients where lymphoma previously demonstrated FDG PET uptake, the absence of such uptake strongly argues against malignant nature of the lesion (Figure 7).

Prominently enhancing lesions are more likely to represent varices, aneurysms, or other highly or completely vascular lesions. Any intraorbital intervention should be conducted as though a vascular lesion is within the differential, and appropriate care should be taken even if not specifically enunciated in the radiologic report.

Management of orbital varices is not standardized; however, these lesions tend to be observed if no significant proptosis, pain, thrombosis, diplopia, or compression of the optic nerve is present. In such cases, surgical intervention is performed; however, the lesions may recur. Our patient's presentation coincided with her heart failure exacerbation
FIGURE 4 Axial T1 Magnetic Resonance Image
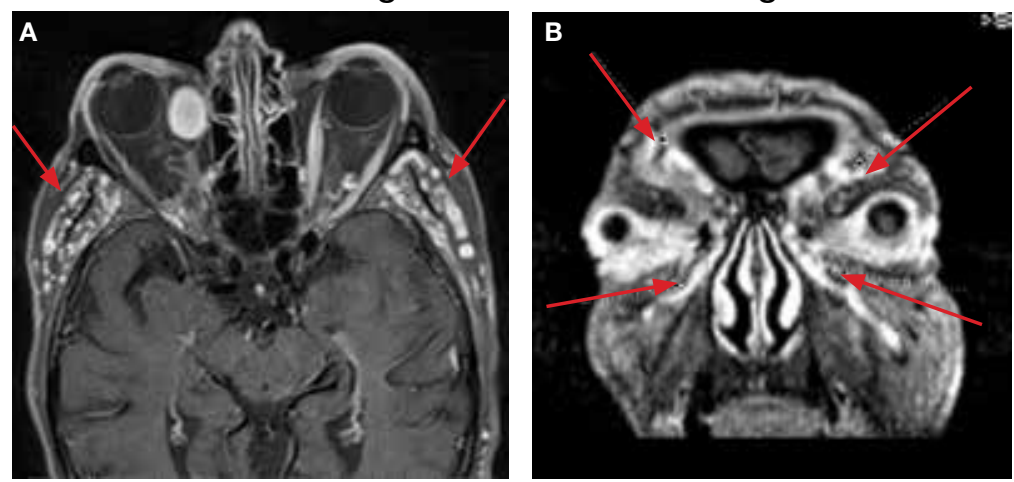

A, weighted postcontrast fat suppressed orbits showing prominent infratemporal vessels (arrows); B, axial T1 magnitization-prepared rapid acquisition and multiple gradient echoes postcontrast coronal reformatted of the brain showing prominent supraorbital and angular veins (arrows).

\section{FIGURE 5 Axial T2 Weighted Fat- Suppressed Magnetic Resonance Image Orbits}

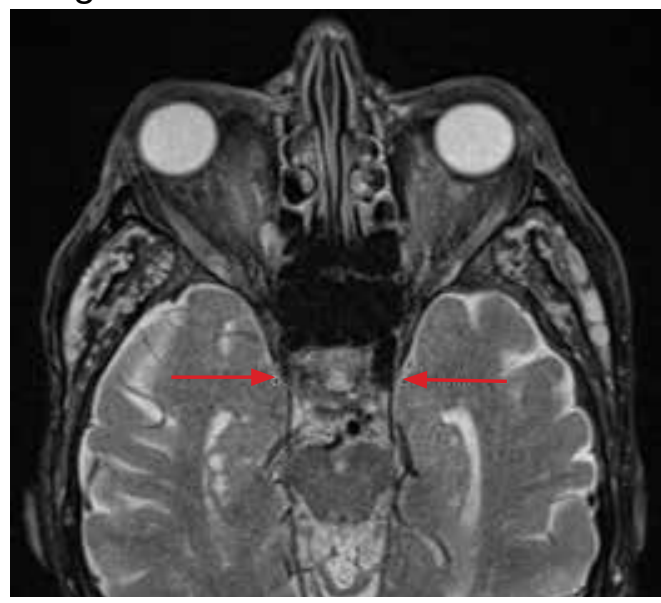

FIGURE 6 Axial T1-Weighted

Postcontrast Fat-Suppressed

Magnetic Resonance Image Orbits Showing Normal Appearing Cavernous Sinus (Arrows)

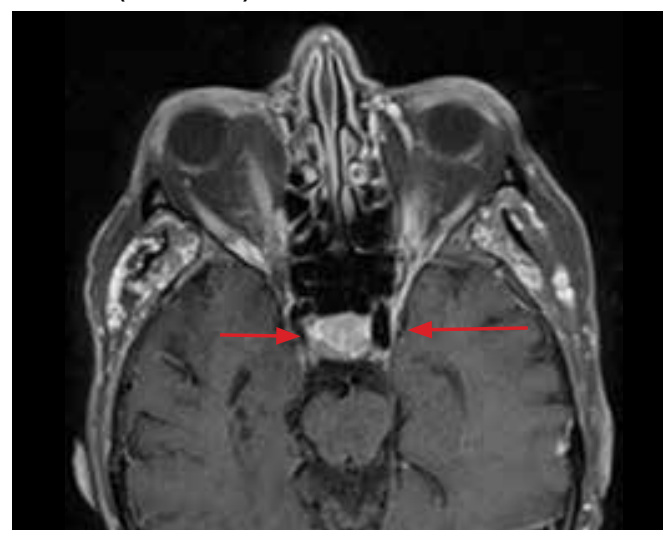




\section{FIGURE 7 Fluorodeoxyglucose-Positron Emission Tomography Without Hypermetabolic Activity Corresponding to the Orbital Lesions (Arrow)}

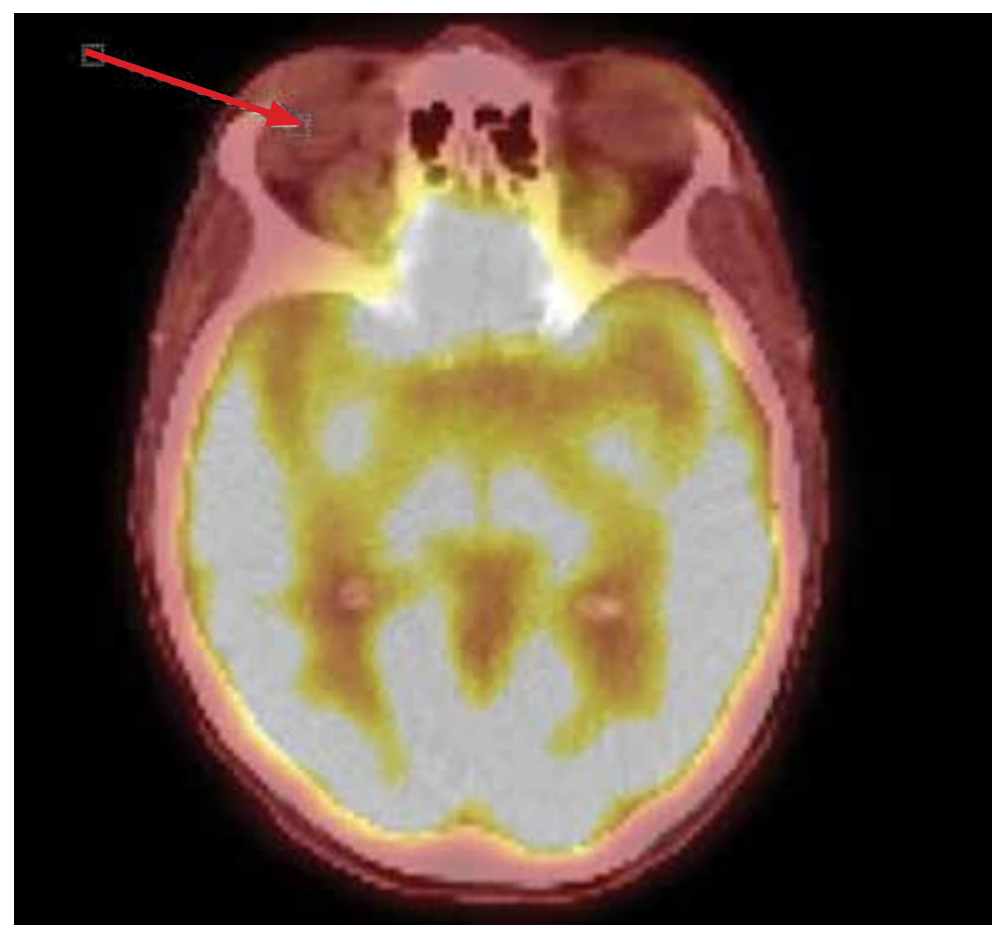

most likely secondary to flow disruption and fluid overload in the venous system, thereby exacerbating her orbital varices. The resolution of our patient's orbital pain in the left orbit was likely due to improved distension after achieving euvolemia after diuresis. In cases where varices are secondary to a correctable etiologies, treatment of these etiologies are in order. Chen and colleagues reported a case of pulsatile proptosis associated with fluid overload in a newly diagnosed case of heart failure secondary to mitral regurgitation. ${ }^{6}$ Thus, orbital pain due to worsened orbital varices may represent a symptom of fluid overload and the provider may look for etiologies of this disease process.

\section{CONCLUSIONS}

We present a case of an orbital varix masquerading as an orbital lymphoma. While the ruling out of a diagnosis that might portend a poor prognosis is always of paramount importance, proper use of investigative studies and a thorough history could have helped elucidate the true diagnosis sooner: In this case an orbital varix masquerading as an orbital lymphoma. Mainly, the use of the Valsalva maneuver during the physical examination (resulting in proptosis) and during radiologic studies might have obviated the need for formal biopsy. Furthermore, orbital pain may be a presenting symptom of fluid overload in patients with a history of orbital varices.

\section{Author disclosures}

The authors report no actual or potential conflicts of interest with regard to this article.

\section{Disclaimer}

The opinions expressed herein are those of the authors and do not necessarily reflect those of Federal Practitioner, Frontline Medical Communications Inc., the US Government, or any of its agencies. This article may discuss unlabeled or investigational use of certain drugs. Please review the complete prescribing information for specific drugs or drug combinations-including indications, contraindications, warnings, and adverse effectsbefore administering pharmacologic therapy to patients.

\section{References}

1. Bacorn C, Gokoffski KK, Lin LK. Clinical correlation recommended: accuracy of clinician versus radiologic interpretation of the imaging of orbital lesions. Orbit. 2021;40(2):133-137. doi:10.1080/01676830.2020.1752742

2. Shams PN, Cugati S, Wells T, Huilgol S, Selva D. Orbital varix thrombosis and review of orbital vascular anomalies in blue rubber bleb nevus syndrome.OphthalmicPlastReconstrSurg.2015;31(4):e82-e86. doi:10.1097/IOP.0000000000000107

3. Islam N, Mireskandari K, Rose GE. Orbital varices and orbital wall defects. Br J Ophthalmol. 2004;88(8):1092-1093.

4. Smoker WR, Gentry LR, Yee NK, Reede DL, Nerad JA. Vascular lesions of the orbit: more than meets the eye. Radiographics. 2008;28(1):185-325. doi:10.1148/rg.281075040

5. Karcioglu ZA, ed. Orbital Tumors. New York; 2005. Chap 13:133-140.

6. Chen $\mathrm{Z}$, Jones $\mathrm{H}$. A case of tricuspid regurgitation and congestive cardiac failure presenting with orbital pulsation. JRSM Cardiovasc Dis. 2012;1(1):cvd.2012.012005. Published 2012 Apr 5. doi:10.1258/cvd.2012.012005 